\title{
BELLE II Pixel Detector
}

\section{Boronat on behalf of the DEPFET coll.*} IFIC (Instituto de Física Corpuscular), Valencia, Spain

E-mail: boronat.arevalo@ific.uv.es

The DEPFET technology is the baseline for the innermost detector of the Belle II experiment at the $\mathrm{e}^{+} \mathrm{e}^{-}$SuperKEKB collider at KEK. This technology integrates signal detection and a first phase of signal amplification in a single silicon structure with a $75 \mu \mathrm{m}$ thin pixel array. This feature provides a very accurate position measurement, with an overall material budget of $0.2 \%$ radiation length and reduces the impact of multiple scattering for tracks with low transversal momentum. Furthermore, the physics goals of the experiment impose challenging requirements to this technology, but DEPFET with its excellent signal-noise-ratio, its lower power consumption and its non destructive readout has proven to be a suitable solution for the Belle II PXD necessities. The vertex pixel detector will consist of two DEPFET layers at radii of $14 \mathrm{~mm}$ and $22 \mathrm{~mm}$ with 8 and 12 modules respectively. The pixel sizes will vary, between $50 \times 50 \rightarrow 55 \mu \mathrm{m}^{2}$ at the fist layer and between $50 \times 70 \rightarrow 85 \mu \mathrm{m}^{2}$ at the second layer, to optimize the charge sharing efficiency. Moreover the four-fold readout in rolling shutter mode provides a readout speed of $20 \mu \mathrm{s} /$ frame. All of these features, the sensor concept and the electronics involved will be presented in detail.

24th International Workshop on Vertex Detector -VERTEX2015-

\section{1-5 June 2015}

Santa Fe, New Mexico, USA

\footnotetext{
* Speaker.
} 


\section{The Belle II PXD.}

The upgrade of Belle detector, Belle II detector, is being constructed at KEK in Tsukuba (Japan) as part of the KEKB collider upgrade (SuperKEKB). This machine is an asymmetric $\mathrm{e}^{-} \mathrm{e}^{+}$ collider, working at $\Psi(\mathrm{nS})$ resonance energies. The expected instantaneous luminosity will be 40 times larger (up to $8 \times 10^{35} \mathrm{~cm}^{2} \mathrm{~s}^{-1}$ ) than its predecessor, aiming an integrated luminosity of $50 \mathrm{ab}^{-1}$. The new accelerator will collide smaller beam sizes, reducing the horizontal and vertical diameter of the beam to the range of nanometers (Nano Beam Concept) and the beam current will increase by a factor of two. This new scenario must cope with higher event rates and background (10 - 20 higher), and accordingly larger radiation damage, occupancy and fake hit production [2].

To deal with the increase of the beam background, the asymmetry at SuperKEKB will be smaller, using electrons with $7 \mathrm{GeV}$ and positrons with $4 \mathrm{GeV}$, compared with its predecessor, which nominally used electrons with $8 \mathrm{GeV}$ and $3.5 \mathrm{GeV}$ for positrons. This will reduce the average separation between the $B$ and $\bar{B}$ decay vertices by a factor of two, down to $100 \mu \mathrm{m}$. To cope with this new scenario, improved vertexing and tracking capabilities are needed for the Belle II detector [3].

\begin{tabular}{c|c}
\hline & Belle II \\
\hline Occupancy & $0.4 \mathrm{hits} / \mu \mathrm{m}^{2} / \mathrm{s}(<3 \%)$ \\
\hline Radiation & $\sim 20 \mathrm{kGy} /$ year \\
\hline Frame time & $20 \mu \mathrm{s}$ (cont r.o. mode) \\
\hline Acceptance & $17^{\circ}-155^{\circ}$ \\
\hline Material budget & $0.21 \% \mathrm{X}_{0}$ per layer \\
\hline Resolution & $15 \mu \mathrm{m}$ \\
\hline
\end{tabular}

Table 1: Belle II PXD requirements [4][3].

The new vertex detector concept will consist on four layers of double-sided silicon strip (SVD) and two layers of highly granular DEPFET pixel detector (PXD). The PXD layers will be located at a radius of $14 \mathrm{~mm}$ and $22 \mathrm{~mm}$ from the interaction point (IP). As a consequence of the low momentum of the particles in the final state, the hit position reconstruction is limited due to the multiple Coulomb scattering, requiring an ultra-thin technology (75 $\mu \mathrm{m}$ in the sensitive area and a $525 \mu \mathrm{m}$ thick rim providing mechanical stability) to keep the material budget below $\sim 0.2 \% X_{0}$ per layer. The spatial resolution needed is $15 \mu \mathrm{m}$. To achieve this resolution and improve the charge sharing between pixels, the pixel sizes will vary from 50x50 $\rightarrow 55 \mu \mathrm{m}^{2}$ (Layer 1) and 50x70 $\rightarrow$ $85 \mu \mathrm{m}^{2}$ (Layer 2). The detector acceptance must cover the range of $17^{\circ}-155^{\circ}$ in azimuth angle [2]. The fast readout will be performed in continuous mode with a time per frame of $20 \mu \mathrm{s}$, keeping the occupancy below 3\% [4]. Additionally, the detector technology has to be radiation hard to cope with the expected $\sim 20 \mathrm{kGy} /$ year (acording similations). 


\section{The DEPFET Module}

\subsection{The DEPFET Pixel}

A DEPFET pixel consists on a field effect transistor (FET) placed on the top of a high resistivity n-type sideward fully depleted silicon bulk. A deep n-doping implantation, the "internal gate", located under the transistor channel (around $1 \mu \mathrm{m}$ below) creates a minimum of potential for the electrons (Figure 1) [1]. The electrons generated by impinging particles will drift towards the surface of the device and will accumulate there. The internal gate is capacitive coupled to the transistor channel, it means that the charge located there, modulates the drain

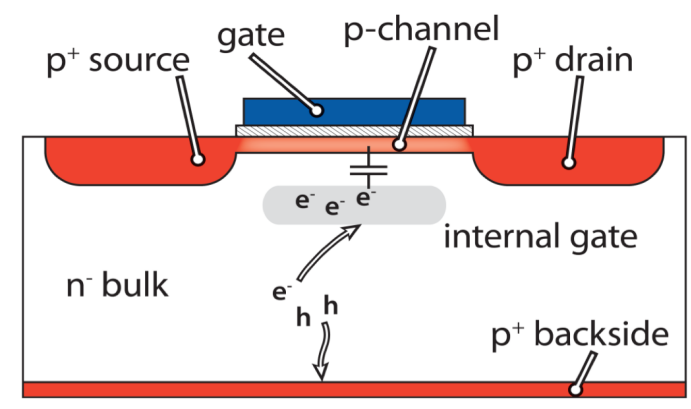

Figure 1: Section of DEPFET Pixel current through the pMOS transistor, which is used as a readout signal. The internal amplification $g_{q}$ is defined as the drain current in $p A$ per electron on the "internal gate". The expected value for the final sensor is of the order of $400 \mathrm{pA} / \mathrm{e}^{-}$ [6]. The readout process is non destructive. The charge located in the "internal gate" remains unaltered. In order to reset the detector and avoid the saturation of the "internal gate", an additional $\mathrm{n}^{+}$ contact is placed in the periphery of each pixel (clear contact). By applying sufficiently high voltage, the clear contact becomes the most attractive potential for the stored electrons, forcing them to drift from the "internal gate" to the clear contact. The potential barrier between the clear contact and the "internal gate" is modulated with an additional polysilicon structure, called cleargate [1].

\subsection{Thinning Process}

In the process of thin down the DEPFET sensor, since the backside is electrically active, conventional thinning techniques, like chemical-mechanical polishing or backside grinding, cannot be used. To thin the sensor down to $75 \mu \mathrm{m}$ in the sensitive area, a special technology has been developed [7]. Two wafers are required, the sensor and the handling wafers. First the backside of the sensor wafer is processed, which is afterwards bonded onto a handling wafer. In the second step the sensor wafer is thinned from top, using conventional methods, down to the desired thickness. Then, all the processing

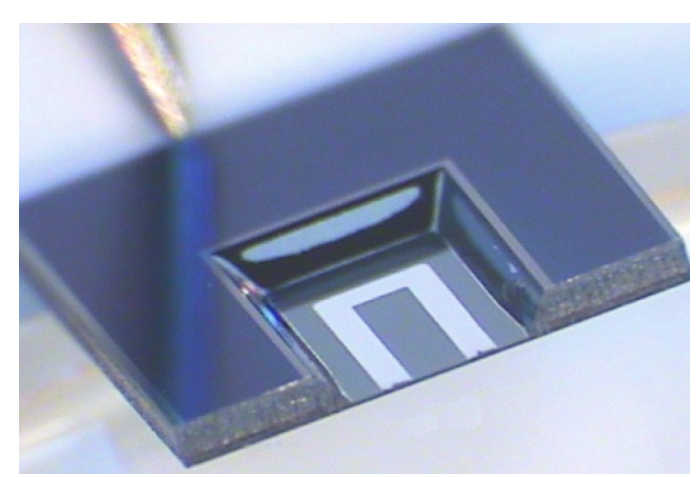

Figure 2: Thinned DEPFET sensor. 
steps on the front side are carried out (implanting, polysilicon deposition, lithography, etc.). Finally, anisotropic etching is used in order to open a window below of the active area of the DEPFET. The handling wafer remains as a frame $(525 \mu \mathrm{m})$ to provide mechanical support to the active area and the balcony to the steering chips (Figure 2). The different contributions to the material budget in the acceptance region are shown in the figure 3.

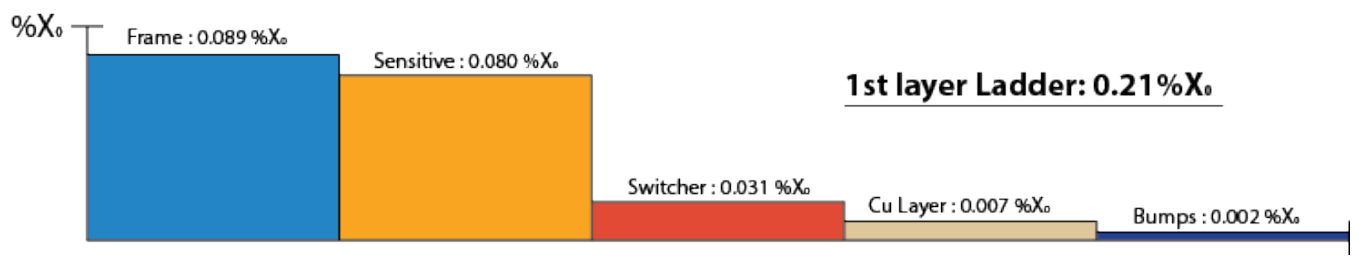

Figure 3: Material Budget Contribution [3]

\subsection{DEPFET half ladder}

Each half-ladder consists of an array of 250x768 pixels. The pixel size depends on the layer location and the expected tilt of the impinging particles. In the first layer, at the center of the ladder, where more perpendicular incidende is expected, to improve the charge sharing, the pixel size will be 50x50 $\mu^{2}$. In the edges, where more tilted incidence is expected, the pixel size will be increased, up to $50 \times 55 \mu m^{2}$. The same strategy is applied to the second layer where the size of the pixels will vary from $50 \times 70 \rightarrow 85 \mu \mathrm{m}^{2}$ [3].

The control ASICs (Switcher) are bump-bonded at the lateral balcony, while the readout ASICs (DCD) and processors

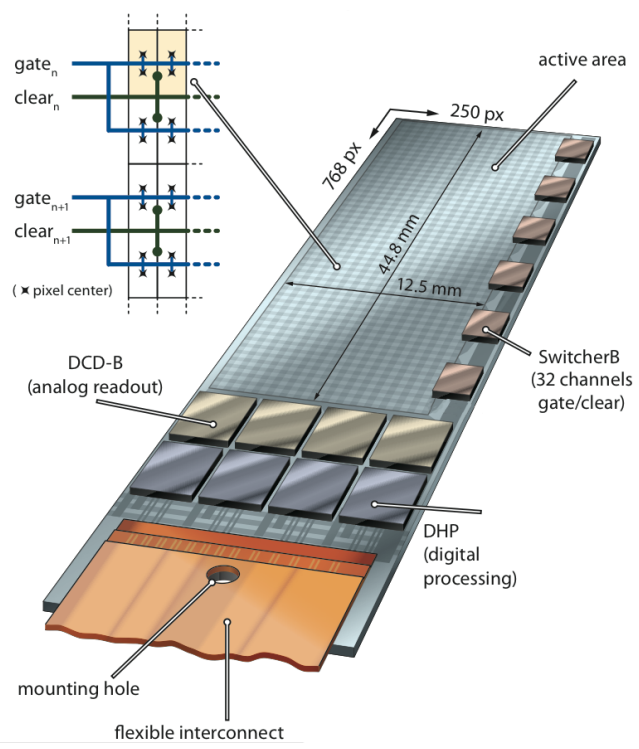

Figure 4: DEPFET half ladder (DHP) are placed at the end of stave (Figure 4), out of the acceptance region. To achieve the required readout speed for Belle II ( $20 \mu \mathrm{m} /$ frame), 4 physical rows are connected to one electrical row, these 192 electrical rows are controlled by six Switchers. Each of the switcher has 32 Gate and Clear outputs for switching the FETs on and off and to apply the Clear pulses [8] [9]. The drain current from the activated transistors go through the drain lines to the current digitizer (DCD-B). Each DCD-B contain 512 ADCs for 256 input channels. It runs at nominal speed of $320 \mathrm{MHz}$ and applies current subtraction and a compensation for pedestal current (2bit DAC). [10] The noise generated is in the order of $50 \mathrm{nA}$. The DCD-B 
produces $\sim 82 \mathrm{Gbps} / \mathrm{s}$ of data and send it to the next ASIC (DHP). The Data Handling Processor (DHP) controls the switcher sequence and the DCDs. Also corrects the data by pedestal, and reduce it, applying a Zero Suppression Cut, to 5Gbps [11]. The data is transferred via a Kapton Flex, using LVDS, to the Data Handling Hybrid (DHE) consisting of a FPGA for further data processing [12].

\subsection{The DEPFET PXD}

A full ladder is constructed with two of these symmetric (with respect to the short edge) half ladders, which are glued together using small ceramic joints $\left(\mathrm{ZnO}_{2}\right)$. The PXD consists of 20 ladders which are arranged cylindrically around the beam pipe in two layers. The inner layer consists in 8 ladders located at $14 \mathrm{~mm}$ of radii and the outer layer consists 12 ladders located at $22 \mathrm{~mm}$ of radii (Table 2).

\begin{tabular}{c|cc}
\hline & Layer 1 & Layer 2 \\
\hline Module & 8 & 12 \\
\hline Radii & $14 \mathrm{~mm}$ & $22 \mathrm{~mm}$ \\
\hline Ladder Size & $15 \times 136 \mathrm{~mm}^{2}$ & $15 \times 170 \mathrm{~mm}^{2}$ \\
\hline Pixel Size & $50 \times 55 \mu \mathrm{m}^{2}$ & $50 \times 70 \mu \mathrm{m}^{2}$ \\
& $50 \times 60 \mu \mathrm{m}^{2}$ & $50 \times 85 \mu \mathrm{m}^{2}$ \\
\hline Pixels & $250 \times 1536$ & $250 \times 1536$ \\
\hline Thickness & $75 \mu \mathrm{m}$ & $75 \mu \mathrm{m}$ \\
\hline
\end{tabular}

Table 2: The Belle II PXD.

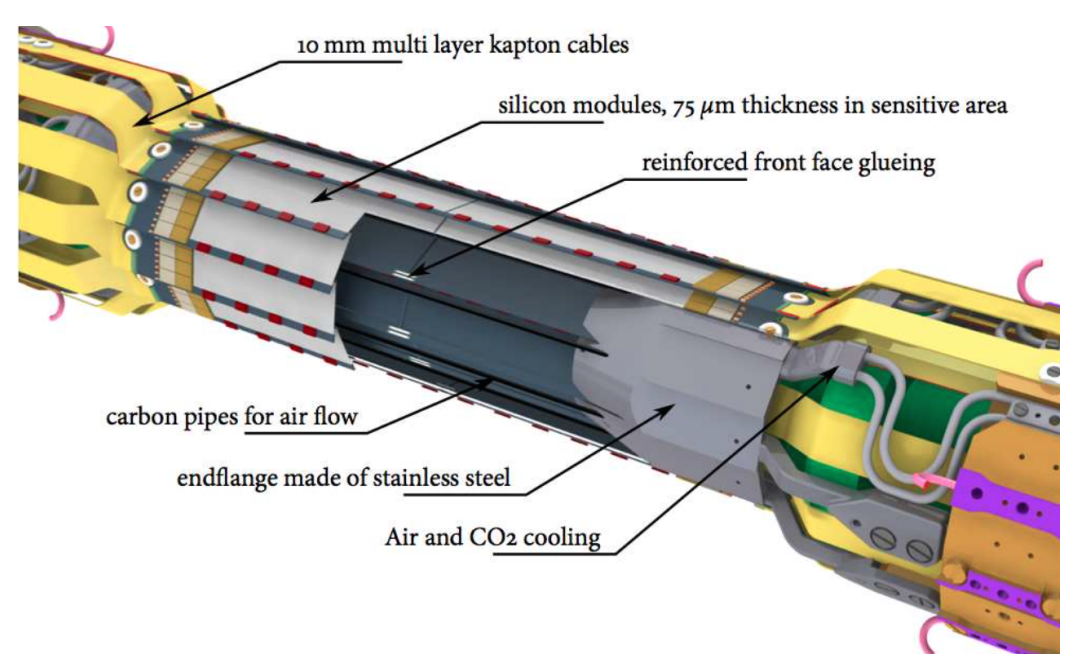

Figure 5: Schematic view of the PXD mounted. 
The ladders are mounted on stainless steel blocks which also serve to dissipate the heat produced by the readout electronics. These blocks have integrated cooling channels to flow cold nitrogen to the sensors and capillaries for a $\mathrm{CO}_{2}$ cooling system. (Figure 6).

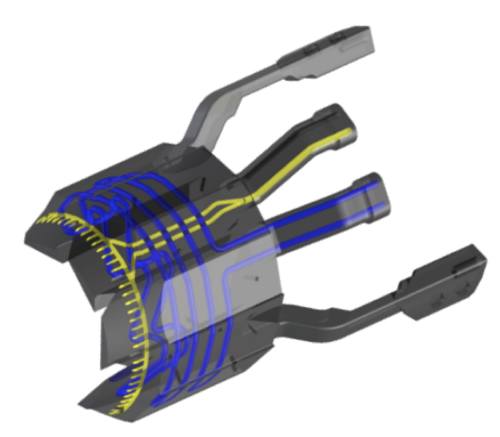

Figure 6: DEPFET half ladder
The total power consumption of one DEPFET module for Belle II is around $9 \mathrm{~W}(0.5 \mathrm{~W}$ the Switchers, $6 \mathrm{~W}$ the DCDs, $2 \mathrm{~W}$ the DHPs and $0.5 \mathrm{~W}$ distributed homogeneously in the active area), adding 360W for the whole PXD detector. Finite element simulation (FEA) and laboratory measurement have proven that, the 2-phase $\mathrm{CO}_{2}$ at $-30^{\circ} \mathrm{C}$ circulating through the stainless steel block is enough to remove the heat produced by the readout electronics. The centre of the ladders has to rely on forced convection with nitrogen flowed from the steel blocks and also using carbon fibers near to the switchers (Figure 5) [13].

\section{DEPFET Performance}

\subsection{Test under beam}

DEPFET technology has been extensively tested in several campaigns of beam test during last years. The usual locations were CERN with pions of $120 \mathrm{GeV}$ and DESY with electrons between 1$6 \mathrm{GeV}$. The main goal of these campaigns is to check all the sensor aspects: charge collection, operation points, efficiency, angular scans, gate length, clear structure, etc. Also, all the system related issues: power supply and DAQ system prototypes. The following results come from the data took with

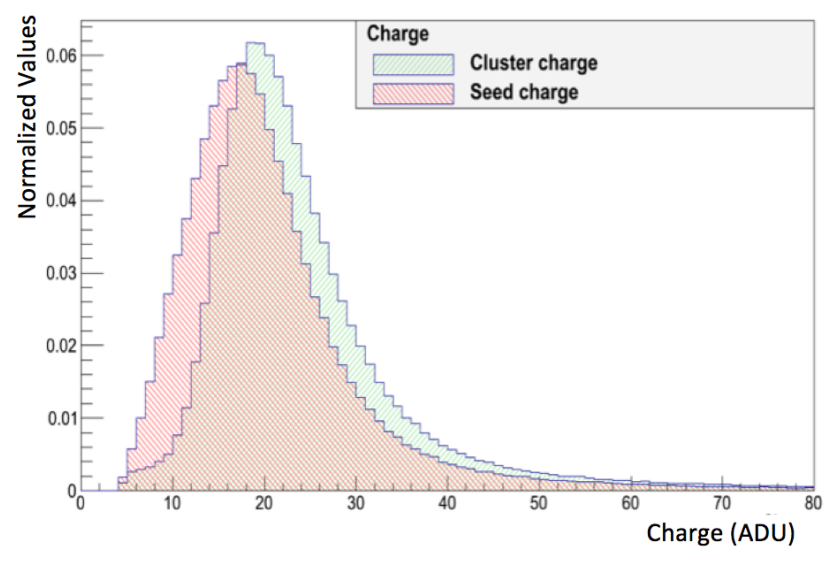

Figure 7: Charge Collection Distribution a PXD6 Belle II design prototype with a pixel size of 50x75 $\mu \mathrm{m}^{2}$ and $50 \mu \mathrm{m}$ thick. The sensor matrix has 32x64 pixels and was operated with one Switcher and one DCD. For this prototype the efficiency of detection was over $99.5 \%$. Figure 7 shows the charge collection distribution. The charge collected by the full clusters featured a most probable value over $23 \mathrm{ADU}$, and considering the intrinsic noise, which is 0.6 
ADU, the signal-to-noise ratio is around 40 .

The sensor resolution is an important parameter that has been studied and optimized, figure 8 shows the resolution (defined as the RMS90) as a function of the zero suppression cut applied. The resolution obtained is $8.5 \mu \mathrm{m}$.

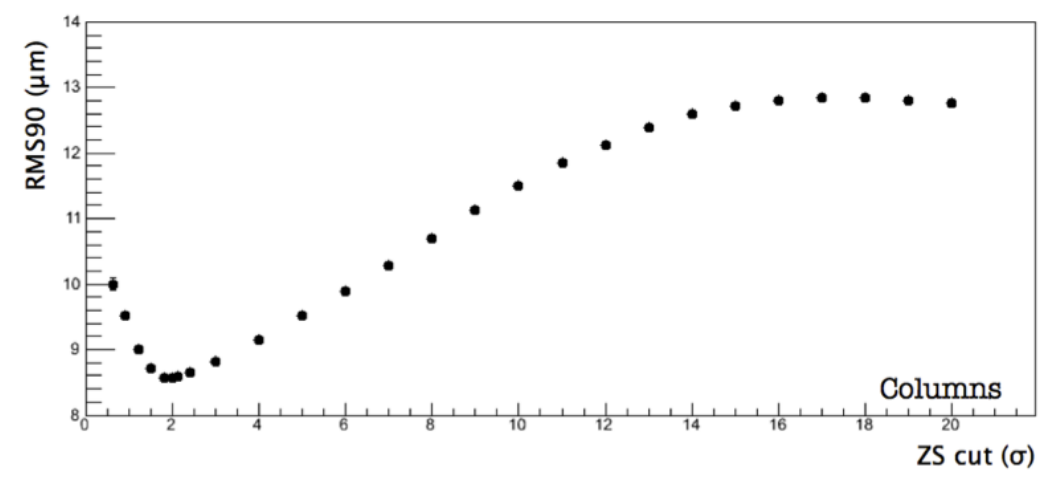

Figure 8: Sensor Resolution as a funciton of the zero suppression cut.

\subsection{Gated Mode}

The SuperKEKB injection scheme foresees an asymmetric electron and positron injection of $25 \mathrm{~Hz}$ resulting in a total injection frequency of $50 \mathrm{~Hz}$. The process of cooling down the new injected bunches by synchrotron radiation will take approximately $4 \mathrm{~ms}$. During this "bunch stabilization" period, part of the particles hit the collimator. The secondary particles created, called as "noisy particle", will cause background occupancy. Consequently, one cannot distinguish whether the signal originates from a real event or from a noisy particle. This would generate a $20 \%$ deadtime, which is not acceptable for a proper operation of the Belle II vertex detector. Hence, further detector investigations are carried out in order to make the detector insensitive for short time periods when noisy bunches are passing. This takes approximately a few hundreds of nanoseconds. During this time, the idea is to make the detector blind. Therefore, the device is switched into the so called "gated mode operation". This is done by applying the Clear pulse and the appropriate Gate voltage (FETs are in the off-state) simultaneously, the potential distribution within the silicon bulk is changed such that newly created electrons within the bulk drift directly to the Clear contact. Meanwhile the amount of stored charge in the internal gate remains untouched, conserving the signal from real Belle II events.

To prove the feasibility of this operation mode, two experiments were performed using a laser to simulate the charge generation in the matrix. In both experiments 8 frames were took consecutively, changing the control sequence to clear the frame when is needed. The first experiment, so called "Junk charge generation", were meant to prove the blindness of the sensor. For this, the laser was pointed in the first frame, to check the matrix response. The clear process is applied after the third frame. The three next frames are operated in gated mode, during this process the laser is 
pointed again. If the sensor is really blind, no signal is expected when normal mode operation is recovered (See figure 9). The second experiment, so called "Signal Charge Restore", is meant to check if the charge is conserved inside the internal gate during the gated mode. The experiment consisted in pointing the laser before gated mode operation and see the remaining charge after it. If charge is conserved a laser spot is expected after gated mode (See figure 10).
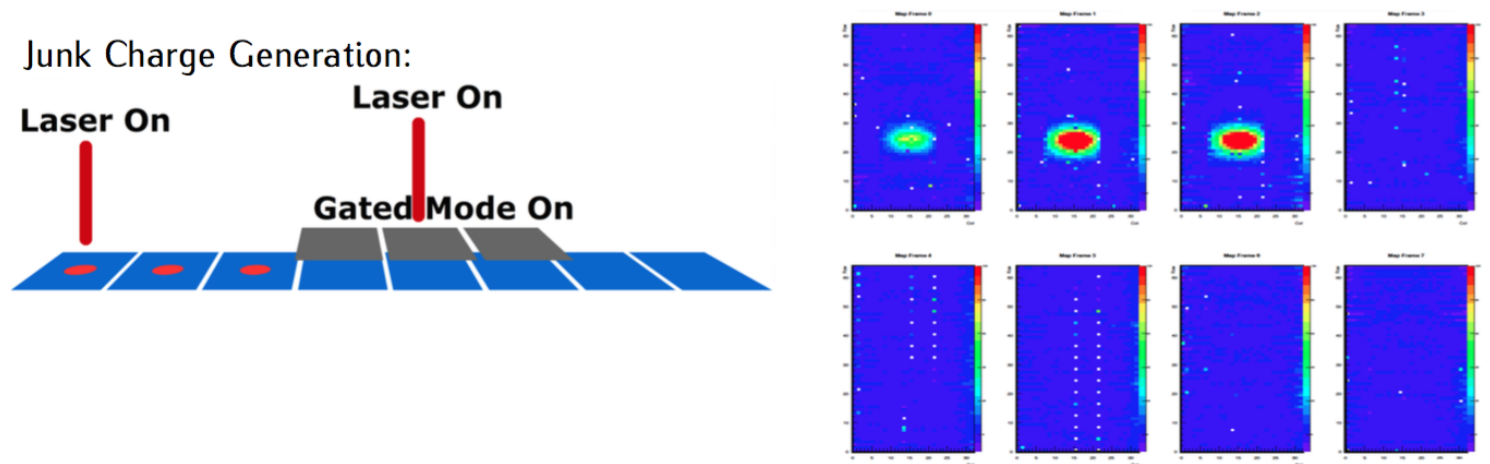

Figure 9: Junk charge generation. Experiment sketch (left), result example (right)
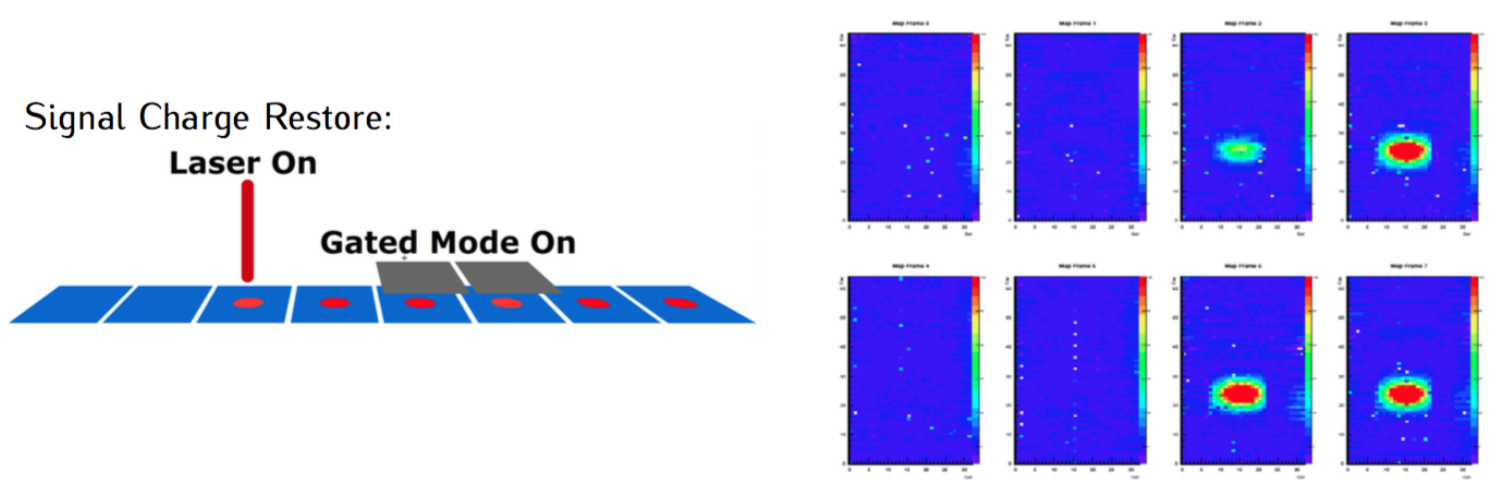

Figure 10: Signal restore process. Experiment sketch (left), result example (right)

\section{Conclusions}

The DEPFET collaboration is developing ultra thin pixel sensors with integrated amplification. The measurements in the laboratory and tests under beam have proved the capability to fulfill all the given challenging requirements in terms of signal-to-noise ratio, material budget, spatial resolution, and readout speed. Also the flexibility of the DEPFET technology to generate a new operational mode (Gated Mode) to avoid the dead time produced during the injection process of SuperKEKB. The pilot run is expected to be ready during summer 2015 , starting the final sprint, which will be finished in spring 2017, when Belle II PXD will become a reality. 


\section{References}

[1] J. Kemmer and G. Lutz, New detector concepts, Nucl. Instrum Meth A253(1987) 365

[2] Belle II Col. Belle II Technical Design Report, arXiv:1012.0352

[3] Carlos Lacasta, The DEPFET pixel detector for the Belle II experiment at SuperKEKB, VERTEX2013 - Proceeding

[4] M. Lemarenko, Test results of the Data Handling Processor for the DEPFET Pixel Vertex Detector, JINST 8 (2013) C01032

[5] M. Boronat, DEPFET pixel detector for future $e^{-} e^{+}$experiments, ICHEP2014 - Proceeding

[6] M. Vos et al, DEPFET active pixel detectors for a future linear $e^{+} e^{-}$collider,arXiv:1212.2160

[7] H. G. Moser, L. Andricek et al, Thinned silicon detetors, Pos(VERTEX2007)013

[8] C. Kreidl, I. Peric, Steering and Readout Chips for the DEPFET sensor matrices, CERN-2007-007

[9] C. Kreidl, Steering electronics, module design and construction of an all silicon DEPFET module, PhD-Thesis, University of Heidelberg (2011)

[10] J. Knopf, P. Fischer, C. Kreidl, I. Peric, A 256 channel 8-bit current digitizer ASIC for the BelleII $P X D, 2011$ JINST 6 C01085

[11] H. Krueger, Front-end electronics for DEPFET pixel detectors at SuperBelle, Nucl. Instrum. Meth. A617 (2010) 337

[12] D. Muenchov et al, The Data Acquisition System of the Belle II Pixel Detector JINST, Instrumentation of colliding beam physics, 2014

[13] P. Ruiz, C. Marinas, The ultra low masss cooling system of the Belle II DEPFET detector, Nucl. Instrum. Meth A 731(2013) 280 